\title{
SIMULAÇÃO NUMÉRICA DE UM CONDENSADOR A AR
}

\author{
R. S. MELLO e A. L. H. COSTA \\ Universidade do Estado do Rio de Janeiro \\ E-mail para contato: rsmello@outlook.com
}

\begin{abstract}
RESUMO - A crescente necessidade da indústria química em aperfeiçoar seus processos criou a necessidade de maior acurácia nos cálculos de engenharia. Com isso, simuladores vêm obtendo grande importância como ferramenta para auxiliar engenheiros e projetistas. O desenvolvimento destas ferramentas capazes de estimar as condições operacionais em trocadores de calor é de extrema importância econômica. Isto se torna mais evidente em condensadores a ar, onde a utilização de ar ambiente para resfriamento em processos requer maior controle das variáveis envolvidas. No presente trabalho, foi desenvolvida a simulação de um modelo matemático de um condensador a ar através das equações de balanço de energia complementadas pela equação da taxa baseada no conjunto de coeficientes de transferência. $\mathrm{O}$ modelo correspondente é solucionado através do método Newton-Raphson. Os resultados da simulação foram obtidos através da aplicação da rotina desenvolvida em um exemplo típico de um condensador a ar.
\end{abstract}

\section{INTRODUÇÃO}

Os trocadores de calor são equipamentos que promovem modificações de temperatura e/ou do estado físico das correntes de processo. Basicamente, esses equipamentos permitem a transferência de calor entre uma corrente quente e uma corrente fria. Existem diferentes tipos de trocadores de calor (casco e tubo, placas, air-cooler, etc.), cada um apresentando um mecanismo diferente de troca térmica (Silva et al., 2005).

Air-coolers são equipamentos que utilizam o ar ambiente para resfriar uma corrente de processo. Esses equipamentos são o segundo tipo de trocador de calor mais utilizado em indústrias químicas e de processamento de petróleo. Em determinadas situações, podem ser mais vantajosos que trocadores de calor casco-e-tubo convencionais, principalmente em locais com suprimento de água restrito, devido a limitações climáticas ou ambientais. Mesmo tendo um custo de instalação mais elevado, seu custo operacional é menor em comparação com outros trocadores de calor (Serth, 2007).

Logo, o conhecimento do comportamento da transferência de calor nesse equipamento é de suma importância na ocorrência de variações na temperatura ambiente, de modo a predizer de que forma a taxa de transferência de calor será afetada por mudanças climáticas (estações do ano, chuva, vento, etc.). Neste contexto, esse trabalho apresenta uma proposta de modelo matemático e a simulação numérica de um condensador refrigerado a ar (air-cooler com condensação). Ao invés de equações integradas, baseadas em coeficientes uniformes, o modelo diferencial proposto é capaz de considerar as variações dos coeficientes de transferência ao longo da área de troca térmica. 


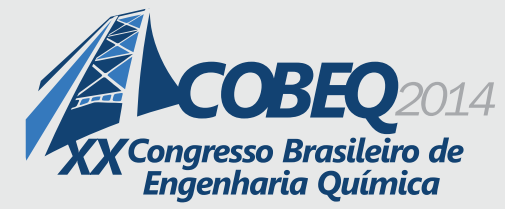

19 a 22 de outubro de 2014

Florianópolis/SC

\section{MODELAGEM DO TROCADOR DE CALOR}

A modelagem de trocadores de calor é composta por dois balanços de energia, aplicados às correntes quente e fria do trocador, e por uma equação de taxa de transferência de calor. A equação diferencial de energia para um fluido escoando pode ser representada por (Incropera et al., 2008):

$$
d H+m(v d v+g d z)=d Q-d W_{e}
$$

onde $H$ é a taxa de entalpia do sistema, $m$ é a vazão mássica, $v$ é a velocidade do fluido, $z$ é a elevação, $Q$ é a taxa de transferência de calor e $W_{e}$ é a taxa de trabalho mecânico. Considerando o escoamento de um fluido ao longo de um trocador de calor, ou seja, não há trabalho mecânico e as variações de energia potencial e cinética são insignificantes quando comparadas com as variações de entalpia, a Equação 1 pode ser simplificada para:

$$
d H=d Q
$$

A Equação 2 pode ter formatos diferentes para um fluido sem mudança de fase (no presente caso, o ar) e para um fluido puro de duas fases (no presente caso, vapor d'água saturado). Estas formas particulares são, respectivamente:

$$
\begin{aligned}
& m C_{p} d T=d Q \\
& m \lambda d \beta=d Q
\end{aligned}
$$

onde $C_{p}$ é a capacidade calorífica do fluído, $\lambda$ é o calor latente do fluido e $\beta$ é a fração de vapor na corrente.

O termo relacionado à taxa de transferência de calor dos balanços de energia (Equações 3 e 4 ) pode ser calculado através do coeficiente global de transferência de calor (desconsiderando a condução axial):

$$
d Q=U d A\left(T_{H}-T_{C}\right)
$$

onde $T_{H}$ e $T_{C}$ são as temperaturas dos fluidos quente e frio e $U$ é o coeficiente global de transferência de calor que, para trocadores de calor do tipo air-cooler, baseado na área externa do tubo, é calculado por:

$$
U=\left(\left(\frac{1}{h_{i}}+R_{f i}\right) \frac{1}{A_{i}}+\frac{\ln \left(D_{e} / D_{i}\right)}{2 \pi k_{t} L}+\frac{R_{f e}}{\eta_{t} A_{e}}+\frac{1}{\eta_{t} h_{e} A_{\ell}}\right)^{-1} \times \frac{1}{A_{e}}
$$

onde $h_{i}$ e $h_{e}$ são os coeficientes de convecção interno e externo, $A_{i}$ e $A_{e}$ são as áreas interna e externa de troca térmica, $R_{f i}$ e $R_{f e}$ são as resistências de depósito interna e externa, $k_{t}$ é a condutividade térmica do tubo e $\eta_{t}$ é a eficiência da superfície aletada. 


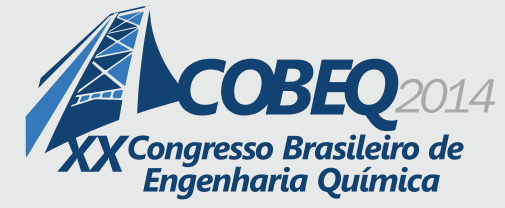

O método de solução proposto para o modelo envolve a discretização do sistema composto pelas Equações 3, 4 e 5 ao longo da área de cada fileira de tubos do trocador de calor, para:

$$
\begin{aligned}
& \left(m_{C} / M\right) C p_{C}\left(T_{C i+1, j}-T_{C i, j}\right)=Q_{i, j+1}-Q_{i, j} \\
& \left(m_{H} / N\right) \lambda\left(\beta_{i, j}-\beta_{i, j+1}\right)=Q_{i, j+1}-Q_{i, j} \\
& Q_{i, j+1}-Q_{i, j}=U_{i, j}\left[T_{H}-\left(T_{C i+1, j}+T_{C i, j}\right) / 2\right] \Delta A
\end{aligned}
$$

onde $i=1, \ldots, N$ corresponde ao índice da fileira e $j=1, \ldots, M$ corresponde ao índice da malha de discretização, $m_{C}$ é a vazão mássica de ar que passa pelo trocador, $m_{H}$ é a vazão de vapor que passa pelo mesmo.

A solução do sistema de equações algébricas não lineares resultante é realizada pelo método Newton-Raphson.

\subsection{Coeficientes de Transferência}

O escoamento de uma mistura de vapor e líquido no interior de um tubo horizontal ocorre de várias formas, de acordo com a velocidade de escoamento e as frações de líquido e vapor presentes no mesmo. A abordagem adotada por este trabalho simplifica essas formas de escoamento, para a avaliação do coeficiente de transferência de calor por convecção no tubo, em apenas duas possibilidades: anular e estratificado. Esta abordagem empregada é baseada na descrição presente em Butterworth (1986).

No caso do escoamento anular, as forças de cisalhamento se sobrepõem às forças gravitacionais e correlações baseadas no escoamento vertical de uma mistura líquido-vapor podem ser aplicadas na avaliação do coeficiente de transferência de calor por convecção nos tubos. Este coeficiente para o escoamento estratificado é calculado baseado nos resultados fornecidos pela teoria de Nusselt sobre condensação em filme ao longo de um tubo. A identificação de que tipo de regime de escoamento prevalece é feita considerando a razão entre as forças de cisalhamento e gravitacional que atuam sobre o fluido. Essas correlações envolvem o uso do valor da temperatura da parede do tubo, que é calculada através de um método iterativo. O coeficiente de transferência de calor por convecção do lado do ar foi avaliado pela correlação de Briggs e Young, de acordo com a descrição presente em Serth (2007).

\section{ESTRUTURA DO PROGRAMA}

As rotinas de simulação dos modelos apresentados neste artigo foram implementadas usando o software Scilab, devido a sua facilidade de resolução de problemas numéricos, e pelo mesmo ser um software aberto. 


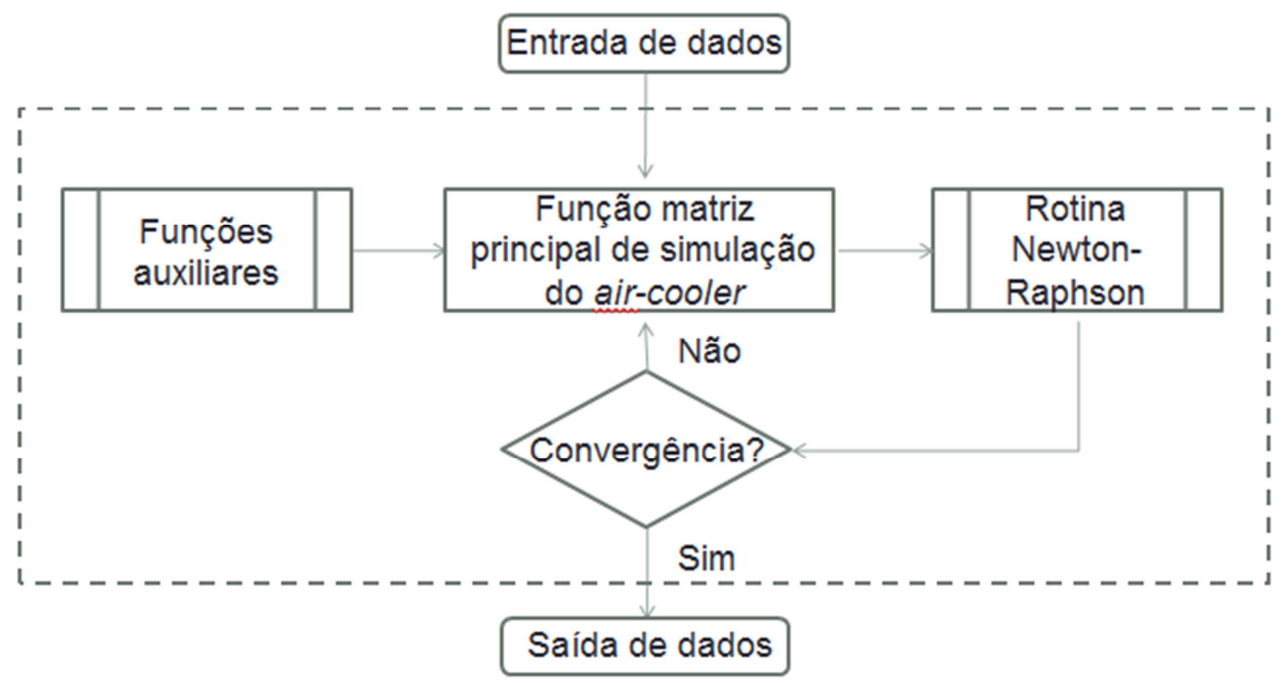

Figura 1 - Estrutura do programa de simulação de condensadores refrigerados a ar.

A Figura 1 sintetiza toda a estrutura do programa proposto. O mesmo é composto por três funções auxiliares, baseadas nos cálculos do coeficiente de convecção interno, do coeficiente de convecção externo e do coeficiente global de transferência de calor; uma função principal, que é composta por um sistema de equações na forma matricial baseado nas Equações 7, 8 e 9; e por uma rotina Newton-Raphson.

\section{RESULTADOS}

\subsection{Teste de Caso}

Um problema de simulação é apresentado neste artigo: o da condensação de uma corrente saturada de acetona por um trocador de calor do tipo air-cooler. Os dados das correntes são apresentados na Tabela 3 e os dados mecânicos do trocador de calor são apresentados na Tabela 4. 
Tabela 3 - Teste de caso: dados da corrente

\begin{tabular}{|c|c|c|c|}
\hline \multicolumn{4}{|c|}{ Dados dos fluidos } \\
\hline & \multicolumn{2}{|c|}{ Acetona } & $\mathrm{Ar}$ \\
\hline & Vapor & Líquido & \\
\hline Vazão mássica (kg/s) & \multicolumn{2}{|c|}{4,126} & 200,00 \\
\hline Temperatura na entrada $\left({ }^{\circ} \mathrm{C}\right)$ & \multicolumn{2}{|c|}{$77^{*}$} & 25 \\
\hline Massa específica $\left(\mathrm{kg} / \mathrm{m}^{3}\right)$ & 3,99 & 719,61 & 1,75 \\
\hline Capacidade térmica (J/kg.K) & - & 2379 & 1003,9 \\
\hline Viscosidade dinâmica (Pa.s) & $9,0 \cdot 10^{-6}$ & $2,04 \cdot 10^{-4}$ & $1,85 \cdot 10^{-5}$ \\
\hline Condutividade térmica (W/m.K) & - & 0,133 & 0,026 \\
\hline Entalpia de vaporização (kJ/kg) & - & 486,8 & - \\
\hline Resistência de depósito $\left(\mathrm{m}^{2} \cdot \mathrm{K} / \mathrm{W}\right)$ & $9,0 \cdot 10^{-5}$ & - & $1,0 \cdot 10^{-5}$ \\
\hline
\end{tabular}

* Temperatura de saturação do fluido quente.

Tabela 3 - Teste de caso: dados do trocador

\begin{tabular}{|l|c|}
\hline \multicolumn{2}{|c|}{ Dados do air-cooler } \\
\hline Comprimento dos tubos $(\mathrm{m})$ & 6,00 \\
\hline Diâmetro externo dos tubos $(\mathrm{m})$ & $3,00 \cdot 10^{-2}$ \\
\hline Diâmetro interno dos tubos $(\mathrm{m})$ & $2,84 \cdot 10^{-2}$ \\
\hline Passo dos tubos $(\mathrm{m})$ & $5,92 \cdot 10^{-2}$ \\
\hline Número de tubos & 210 \\
\hline Número de fila de tubos & 4 \\
\hline Largura dos feixes de tubos (m) & 5,00 \\
\hline Número de aletas por metro de tubo & 197 \\
\hline Diâmetro externo das aletas (m) & $6,2 \cdot 10^{-2}$ \\
\hline Espessura das aletas (m) & $5,8 \cdot 10^{-4}$ \\
\hline Condutividade do material (W/m.K) & 500 \\
\hline
\end{tabular}

\subsection{Resultados da Simulação}

As Figuras 2 e 3 retratam a variação da fração de vapor de acetona e do fluxo de transferência de calor ao longo do comprimento do tubo para cada fileira do trocador de calor. A fração de vapor que sai do condensador é de 0,81 . A Figura 4 demostra a variação da temperatura de saída do ar ao longo da fileira de tubos do air-cooler. 


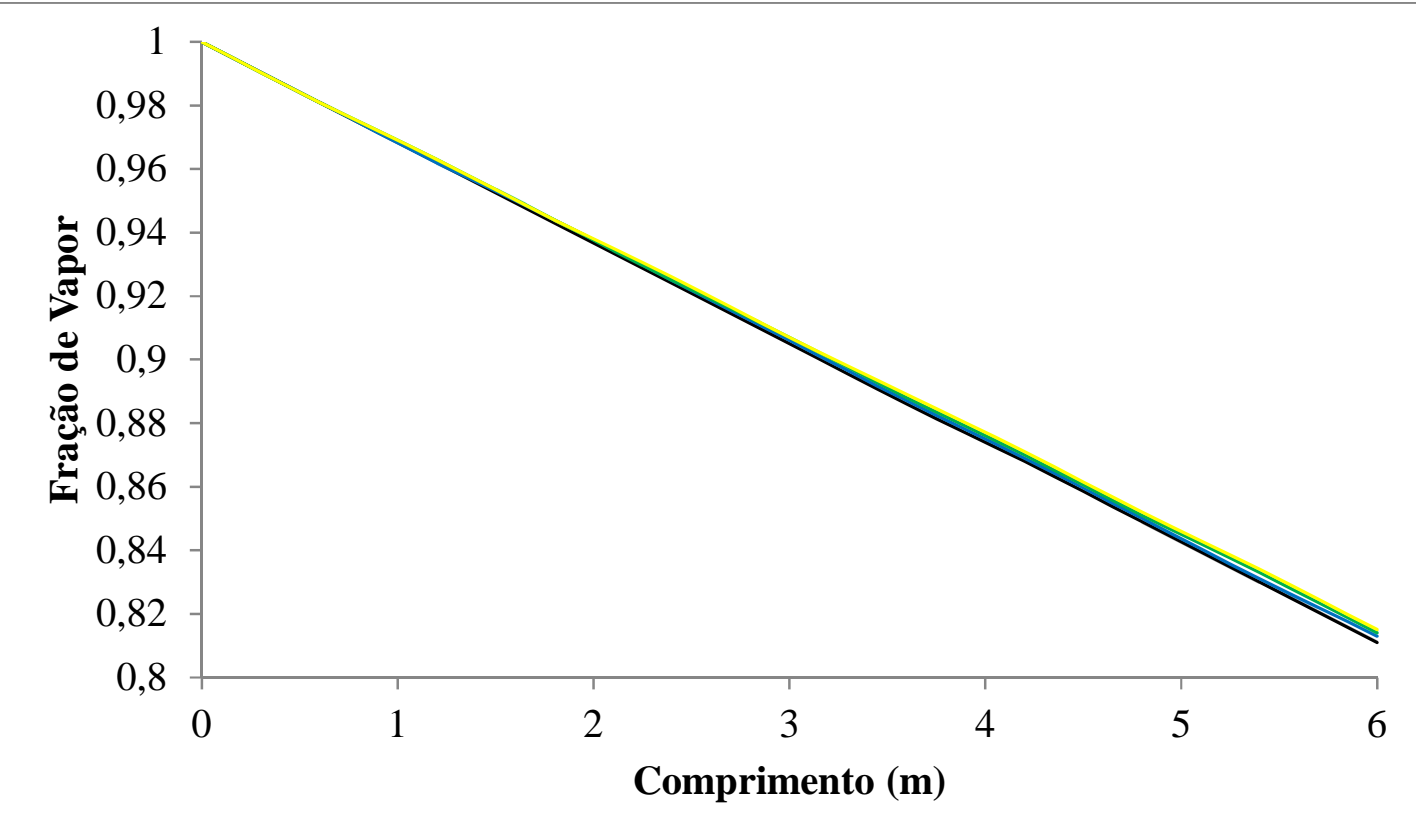

- Fileira 1 -Fileira $2-$ Fileira $3 \longrightarrow$ Fileira 4

Figura 2 - Fração de vapor de acetona ao longo do trocador de calor.

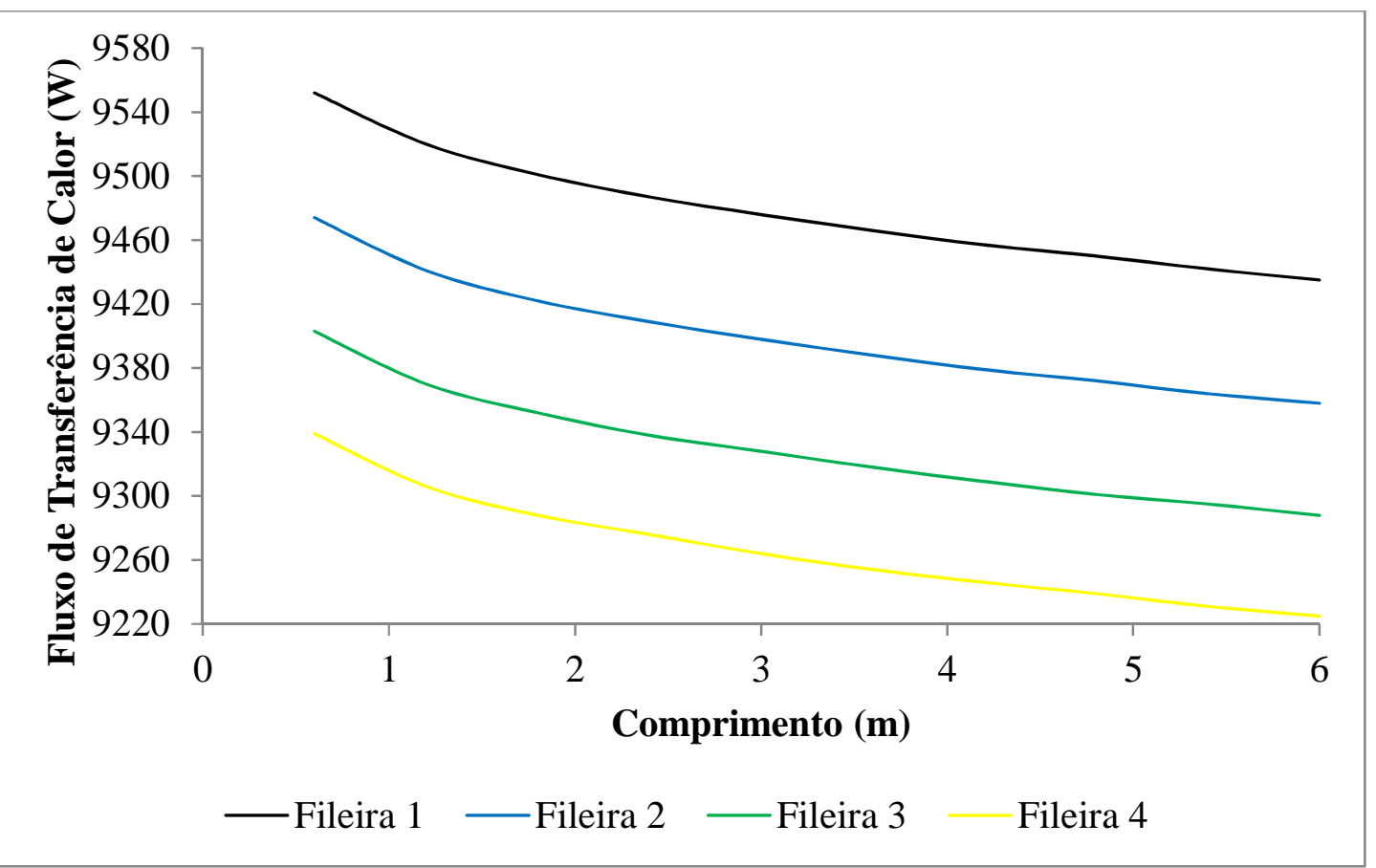

Figura 3 - Fluxo de transferência de calor ao longo do trocador. 


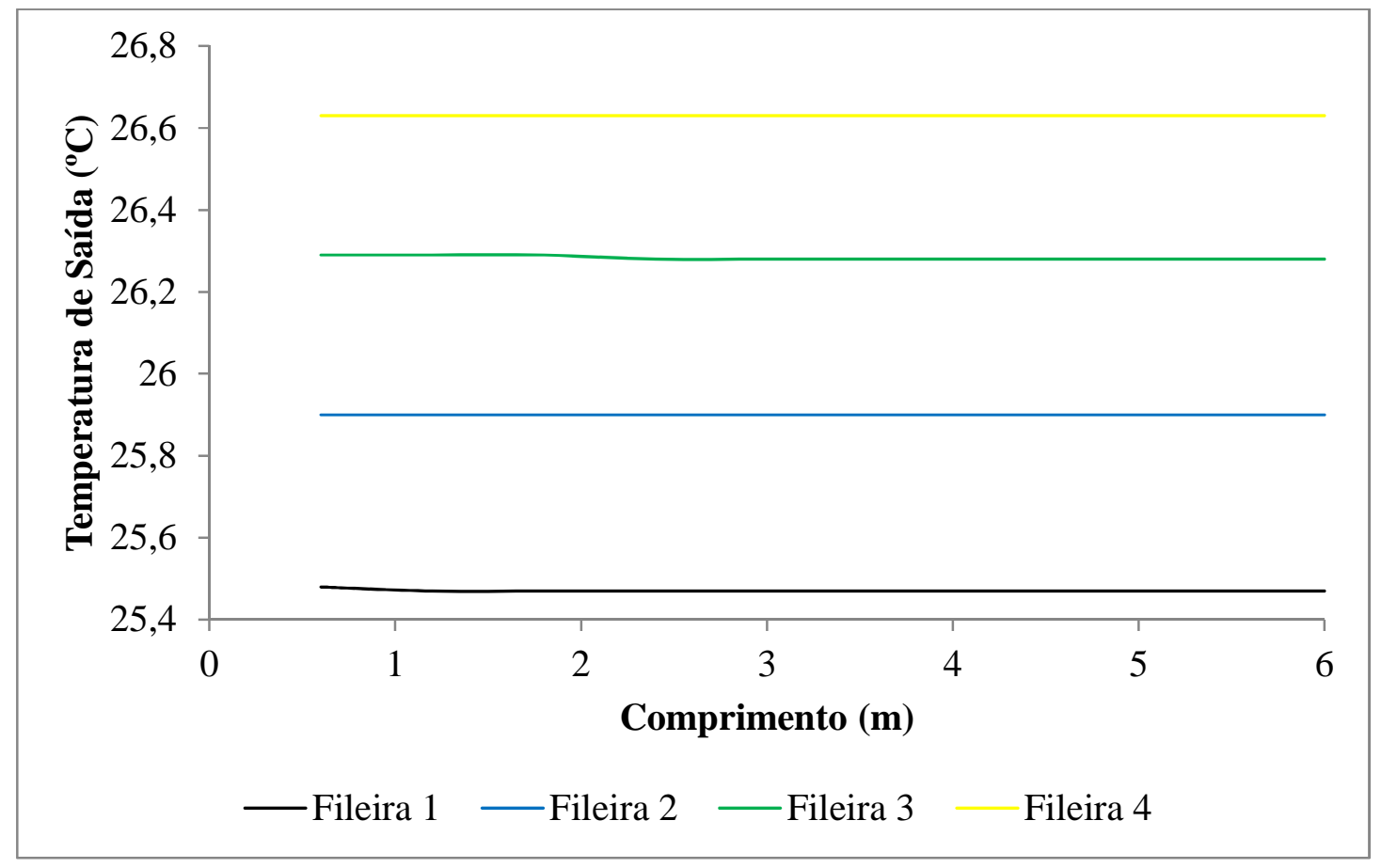

Figura 4 - Perfil da temperatura de saída do fluido frio (ar) do trocador.

Analisando os resultados gerados pela simulação, é possível observar que os mesmos estão coerentes com o comportamento físico esperado. Na Figura 2, observa-se que a fração de vapor diminuiu ao longo do comprimento do trocador em função da progressão da transferência de calor. Não foi possível observar diferenças significativas de variação da fração entre as filas de tubos inferiores e superiores, indicando que a variação da temperatura do ar ao longo do air-cooler não foi significativa a ponto de alterar a condensação nas diferentes fileiras deste.

$\mathrm{Na}$ Figura 3, percebe-se que o fluxo térmico nas filas superiores é menor que o das inferiores, o que é explicado pelo aumento da temperatura do ar nessas filas, implicando na redução do approach nas mesmas. Em uma mesma fila, o fluxo diminuí ao longo do comprimento do trocador em função do aumento da vazão de condensado formado levar a uma redução do coeficiente de convecção. Já na Figura 4, observa-se o aumento da temperatura do ar ao longo das filas do trocador em função da transferência de calor.

\section{CONCLUSÕES}

Este artigo discutiu a elaboração de um modelo matemático e a elaboração de um programa para simulação de trocadores do tipo air-cooler com condensação do fluido quente. O modelo envolve balanços de energia dos fluidos quente e frio, que são resolvidos baseados na discretização dessas equações, resultando em um sistema de equações algébricas não lineares. Os códigos de simulação incluem somente casos de condensação de fluido puro no interior dos tubos do air-cooler. Futuros avanços no algoritmo em questão podem envolver a implementação de uma metodologia envolvendo 
a condensação de misturas multicomponentes.

\section{REFERÊNCIAS}

BUTTERWORTH, D. Film Condensation of Pure Vapor in Heat Exchanger Design Handbook, organized by E.U. Schlünder, EUA: Hemisphere Publishing Corporation, 1983.

INCROPERA, F. P.; DEWITT, D. P.; BERGMAN, T. L.; LAVINE, A. S. Fundamentos de Transferência de Calor e de Massa. Rio de Janeiro: LTC - Livros Técnicos e Científicos Editora S.A., 2008.

MUELLER, A. C. Condensers in Heat Exchanger Design Handbook, organized by E.U. Schlünder, EUA: Hemisphere Publishing Corporation, 1983.

SERTH, R. W. Process Heat Transfer: Principles and Applications, OXFORD: Academic Press, 2007.

SILVA, J. P.; COSTA, A. L. H.; PAREDES, M. L. L.; QUEIROZ, E. M. Modeling and Simulation of Heat Exchangers. Anais do 2nd Mercosur Congress on Chemical Engineering and 4th Mercosur Congress on Process Systems Engineering, v. 1, 2005. 